\title{
Article \\ Optogenetic Manipulation of Olfactory Responses in Transgenic Zebrafish: A Neurobiological and Behavioral Study
}

\author{
Yun-Mi Jeong ${ }^{1,2}$, Tae-Ik Choi ${ }^{1}$, Kyu-Seok Hwang ${ }^{1}{ }^{\oplus}$, Jeong-Soo Lee ${ }^{2}$, Robert Gerlai ${ }^{3, *}$ and Cheol-Hee Kim ${ }^{1, *(D)}$ \\ 1 Department of Biology, Chungnam National University, Daejeon 34134, Korea; angdoym@kribb.re.kr (Y.-M.J.) \\ c860523@naver.com (T.-I.C.); kshwang@krict.re.kr (K.-S.H.) \\ 2 Disease Target Structure Research Center, Korean Research Institute of Biosciences and Biotechnology, \\ Daejeon 34141, Korea; jeongsoo@kribb.re.kr \\ 3 Department of Psychology, University of Toronto Mississauga, Mississauga, ON L5L 1C6, Canada \\ * $\quad$ Correspondence: robert.gerlai@utoronto.ca (R.G.); zebrakim@cnu.ac.kr (C.-H.K.)
}

Citation: Jeong, Y.-M.; Choi, T.-I.; Hwang, K.-S.; Lee, J.-S.; Gerlai, R.; Kim, C.-H. Optogenetic Manipulation of Olfactory Responses in Transgenic Zebrafish: A Neurobiological and Behavioral Study. Int. J. Mol. Sci. 2021, 22, 7191. https://doi.org/ $10.3390 /$ ijms22137191

Academic Editor: Antonino Germana

Received: 10 June 2021

Accepted: 1 July 2021

Published: 3 July 2021

Publisher's Note: MDPI stays neutral with regard to jurisdictional claims in published maps and institutional affiliations.

Copyright: (c) 2021 by the authors. Licensee MDPI, Basel, Switzerland. This article is an open access article distributed under the terms and conditions of the Creative Commons Attribution (CC BY) license (https:/ / creativecommons.org/licenses/by/ $4.0 /)$.

\begin{abstract}
Olfaction is an important neural system for survival and fundamental behaviors such as predator avoidance, food finding, memory formation, reproduction, and social communication. However, the neural circuits and pathways associated with the olfactory system in various behaviors are not fully understood. Recent advances in optogenetics, high-resolution in vivo imaging, and reconstructions of neuronal circuits have created new opportunities to understand such neural circuits. Here, we generated a transgenic zebrafish to manipulate olfactory signal optically, expressing the Channelrhodopsin (ChR2) under the control of the olfactory specific promoter, omp. We observed light-induced neuronal activity of olfactory system in the transgenic fish by examining $c$-fos expression, and a calcium indicator suggesting that blue light stimulation caused activation of olfactory neurons in a non-invasive manner. To examine whether the photo-activation of olfactory sensory neurons affect behavior of zebrafish larvae, we devised a behavioral choice paradigm and tested how zebrafish larvae choose between two conflicting sensory cues, an aversive odor or the naturally preferred phototaxis. We found that when the conflicting cues (the preferred light and aversive odor) were presented together simultaneously, zebrafish larvae swam away from the aversive odor. However, the transgenic fish with photo-activation were insensitive to the aversive odor and exhibited olfactory desensitization upon optical stimulation of ChR2. These results show that an aversive olfactory stimulus can override phototaxis, and that olfaction is important in decision making in zebrafish. This new transgenic model will be useful for the analysis of olfaction related behaviors and for the dissection of underlying neural circuits.
\end{abstract}

Keywords: zebrafish; olfaction; optogenetics; channelrhodopsin

\section{Introduction}

Multimodal integration of various sensory information in the environment is important to generate appropriate behavioral responses. How the brain transforms sensory information into an observable behavioral output, i.e., how a decision is made to respond to a stimulus, is a major question in neuroscience. When two sensory inputs conflict, one sensory influence the other and this dominance may be beneficial in decision making of animals [1]. Auditory information dominates visual cue in certain circumstances [1], and olfactory stimuli often modulates visual perception [2]. However, when incompatible sensory inputs such as an attractive and an aversive cue were given concurrently, which cue would be dominance and the underlying mechanism remains unclear. Olfaction (i.e., sense of smell) is one of the most important senses affecting decision making for survival and fundamental behaviors of animals [3].

Zebrafish is an attractive vertebrate model to study sensory processing in the nervous system for numerous reasons, one of which is that this species possesses a complex behavioral repertoire and exhibits behavioral responses that can be induced reliably [3]. The 
olfactory organ of fish is well developed to perceive odorants in an aquatic environment [4]. Recognition of odors from spoiled food or predators can be important to elicit suitable avoidance behaviors for survival of animal [5]. Zebrafish larvae also respond to visual stimuli. For example, zebrafish larvae show phototaxis whereby the fish swim toward the light [6]. Furthermore, zebrafish larvae spend more time in brightly illuminated areas when placed in a light/dark choice task, a response that is a matter of controversy in adults [7-12]. Along these lines, zebrafish larvae also exhibit complex behavioral responses including spontaneous motor behavior as well as learning and memory [13,14].

Optogenetics is an emerging field that combines genetics and systemic stimulation with a specific wavelength of light to achieve precise temporal control over gain or lossof-function within specific cells of living organisms [15-17]. For example, microbial opsin genes can be introduced to control action potential in specific neuronal populations within freely moving mammals or other intact animal model system. Nagel and colleagues employed channelrhodopsin-2 (ChR2), which is a light-gated ion channel, in excitable cells of the nematode to trigger specific behaviors by blue light $(480 \mathrm{~nm})$, and in zebrafish, photo-activation of $\mathrm{ChR} 2$ in the somatosensory neurons were reported to trigger escape behavior $[18,19]$.

In the current study, we generated a transgenic zebrafish that enables us to control perception of olfactory stimuli. For this, we used optogenetic molecular tools such as ChR2 and Kaede, which is a photo-convertible fluorescent protein. We generated a transgenic fish to stimulate the olfactory sensory neurons with blue light using these optogenetic molecules, ChR2 and Kaede. We made a fusion protein of ChR2 and Kaede, and expressed this construct in zebrafish olfactory sensory neurons under the control of omp (olfactory marker protein) promoter. We analyzed the neuronal activity of olfactory system by blue light using an immediate early gene, $c-f o s$, a marker for neuronal activity, as well as by using a genetically encoded calcium indicator, GCaMP in the transgenic zebrafish.

To examine whether the photoactivation of olfactory sensory neurons affect zebrafish behavior and to investigate which sensory modality, vision or olfaction, dominates decisionmaking, we designed a behavioral paradigm in which the innate phototaxis of zebrafish larvae and the presence of an aversive odorant are conflicted. We found that the wild type zebrafish avoided the arm with the odor stimulus despite that this arm was well illuminated. However, the transgenic zebrafish were insensitive to the aversive odorant when stimulated by the ChR2 specific blue light. These results suggest that olfaction is a critical sensory input in decision making in wild type larval zebrafish as it is able to override phototaxis, an olfaction driven response that is abolished in transgenic fish upon optogenetic activation of the olfactory sensory neurons with the ChR2 specific blue light. These results indicate that the transgenic fish will be useful to investigate the functional analysis of olfactory circuits or related behavior more easily.

\section{Results}

2.1. Establishment and Characterization of Transgenic Zebrafish Expressing Channelrhodopsin-2 in Olfactory Sensory Neurons

Channelrhodopsins (ChR) transport various ions over the membrane in response to light and have been used to manipulate specified neuronal activity and behavior in various model animals [18-20]. We generated a transgenic zebrafish line carrying the omp promoter-driven ChR2-Kaede fusion gene to control neuronal activity of olfactory sensory neurons (OSNs) by blue light (Figure 1A,B). Olfactory marker protein, encoded by omp gene, is specifically expressed in the vertebrate olfactory sensory neurons [21]. We used the upstream promoter and $3^{\prime}$-untranslated region (3'-UTR) of the zebrafish omp gene [22] and newly generated a fusion protein of ChR2 with Kaede. The latter is a fluorescent protein and has property of photo-conversion from green to red by ultraviolet (UV) light $[23,24]$. Subsequently, we generated a transgenic zebrafish by microinjecting plasmid DNA and Tol2 transposase mRNA into fertilized eggs (Figure 1A, Figures S1 and S2). We wanted to activate one side of the same embryo. The other side of the embryo is used as an internal 
control. We fused Kaede to mark a region of light irradiation because the Kaede protein converted green to red upon light delivery as employed before [25].

A

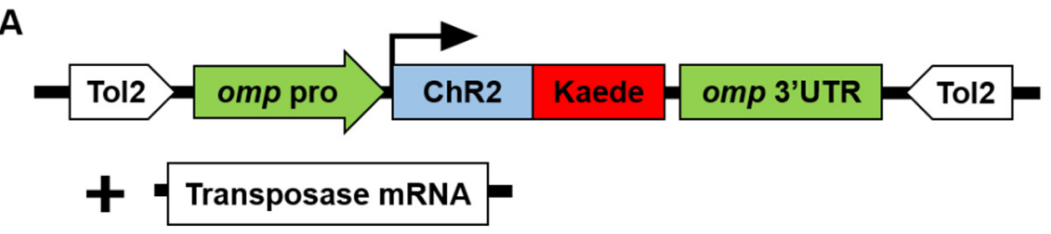

B
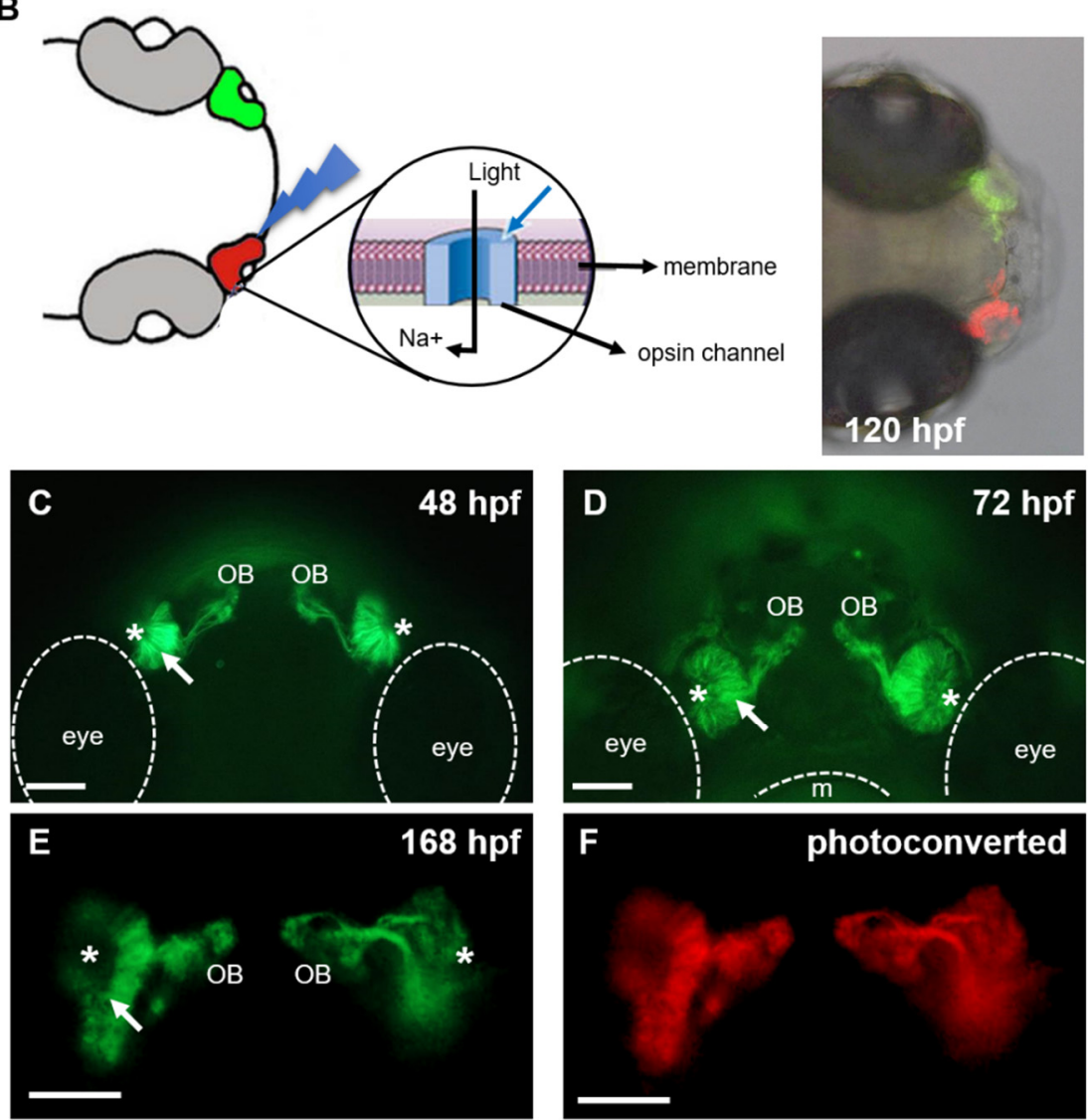

Tg(omp:ChR2-Kaede)

Figure 1. Generation of a transgenic zebrafish expressing ChR2-Kaede in olfactory sensory neurons. (A) Generation of transgenic fish using the expression construct of omp-driven ChR2-Kaede with transposase mRNA. (B) Schematic view of the transgenic zebrafish expressing channelrhodopsin-2 (ChR2) in olfactory sensory neurons. The green fluorescence of Kaede was photo-converted to red color, as an indicator for activation. (C,D) Ventral view of the head of the transgenic zebrafish embryos at $48 \mathrm{hpf}$ and $72 \mathrm{hpf}$. (E,F) Dorsal view of the transgenic fish at $168 \mathrm{hpf}$. The Kaede protein of OSNs was photoconverted by UV light green to red fluorescence (F). Asterisks indicate the nasal pit, and arrows indicate the olfactory epithelium. m, mouth; OB, olfactory bulb. Scale bars, $50 \mu \mathrm{m}$.

The fluorescence of the ChR2-Kaede fusion protein in transgenic zebrafish fish first appeared in the olfactory epithelium at $48 \mathrm{~h}$ post fertilization (hpf). The olfactory sensory neurons projected their axons toward the developing brain, and their axon terminals were observed mainly around the developing olfactory bulb (OB) at $48 \mathrm{hpf}$ (Figure 1C). At $72 \mathrm{hpf}$, the olfactory epithelium and nasal pit became expanded and olfactory nerve bundles were thickened (Figure 1D). ChR2-Keade expression in olfactory sensory neurons was observed by $168 \mathrm{hpf}$ (Figure 1E) and continued to adulthood (data not shown). By UV photo-activation, the green fluorescence of Kaede was photo-converted to red color 
(Figure 1E,F). Kaede is used as reporter to visualize olfactory sensory neurons and to mark regions excited by light.

\subsection{Evaluation of Light Induced Neuronal Activity Using Immediate Early Gene in the Transgenic Fish}

To evaluate ChR2 specific blue light-evoked neuronal activity of olfactory region in the newly generated transgenic fish, we quantified transcriptional activity of an immediate early gene, $c$-fos. Immediate early genes (IEGs) respond rapidly and transiently to a variety of cellular stimuli like neuronal depolarization [26-28]. Odor evoked neuronal activity using $c$-fos in rat was also reported [29,30]. In the absence of sensory stimulation, the brain generally expresses low basal levels of IEGs. We performed whole-mount in situ hybridization to analyze mRNA levels of $c$-fos after blue light irradiation at olfactory organ instead of an odor stimulus. ChR2 specific blue light was irradiated at the olfactory organ of 5 day old wild type and transgenic (TG) zebrafish larvae for $5 \mathrm{~s}$. Thirty minutes after the irradiation the subjects were fixed. c-fos expression was ectopically induced in the olfactory epithelium of light-irradiated transgenic fish (Figure 2D). In contrast, blue light irradiated wild type and non-irradiated transgenic and wild type fish showed basal level of $c$-fos expression in the olfactory epithelium (Figure 2A-C). The intensity of $c$-fos transcript in the region of olfactory epithelium was measured using Image J software (Figure 2E). The intensity in the blue light-irradiated transgenic fish was higher than non-irradiated transgenic fish and light-irradiated wild-type fish. These results indi-cate that optical stimulation in the transgenic fish elicited neuronal activity in the olfactory epithelium. Since ChR2 responds to blue light of approximately $470 \mathrm{~nm}$ wavelength [15], we performed whole-mount in situ hybridization in the transgenic fish under various light conditions to confirm that the blue light-evoked $c$-fos expression in the transgenic fish was indeed due to ChR2. Under the blue ( $480 \mathrm{~nm}), \mathrm{UV}(420 \mathrm{~nm})$ and yellow $(610 \mathrm{~nm})$ light sources on a dissecting microscope, the transgenic zebrafish were irradiated and then fixed. In the siblings, expression of $c$-fos remained at basal level under these light conditions (Figure S3). Transgenic fish, however, irradiated with blue or UV light source showed induction of $c$-fos in olfactory epithelium and olfactory bulb. On the other hand, the yellow light irradiated transgenic fish displayed basal level of $c$-fos expression, similar to non-transgenic control fish (Figure S3).

\subsection{Inhibition of Light-Evoked Activity by GABA Agonist in Transgenic Fish}

GABA (gamma-aminobutyric acid) is the primary inhibitory neuro-transmitter and leads to a hyperpolarization of target neuron in most vertebrate brain areas. GABAergic interneurons in the olfactory bulb of vertebrates play crucial roles in the processing of odor-evoked patterns. In zebrafish, the application of GABA (5-10 mM) suppressed odor responses in the developing olfactory bulb [31,32]. To examine whether GABAergic activation suppresses the ChR2 specific blue light-evoked odor responses in the transgenic fish, we analyzed the expression level of $c-f o s$ after GABA administration in the transgenic zebrafish as described before [31]. In the ChR2 specific blue light-irradiated transgenic fish, transcripts of $c$-fos were detected at the olfactory epithelium (Figure 2H). In contrast, in the ChR2 specific blue light-irradiated transgenic fish that were administered $7 \mathrm{mM}$ GABA for $1 \mathrm{~h}$, the area where $c$-fos positive neurons were found was reduced in the olfactory bulb as well as in olfactory epithelium compared to control (Figure 2G,I). Only GABA treated (no ChR2 specific blue light) transgenic fish showed normal basal level of $c$-fos expression in the olfactory epithelium (Figure 2F). These results showed that GABA could inhibit the $\mathrm{ChR} 2$ specific blue light evoked odor responses in the transgenic fish. 

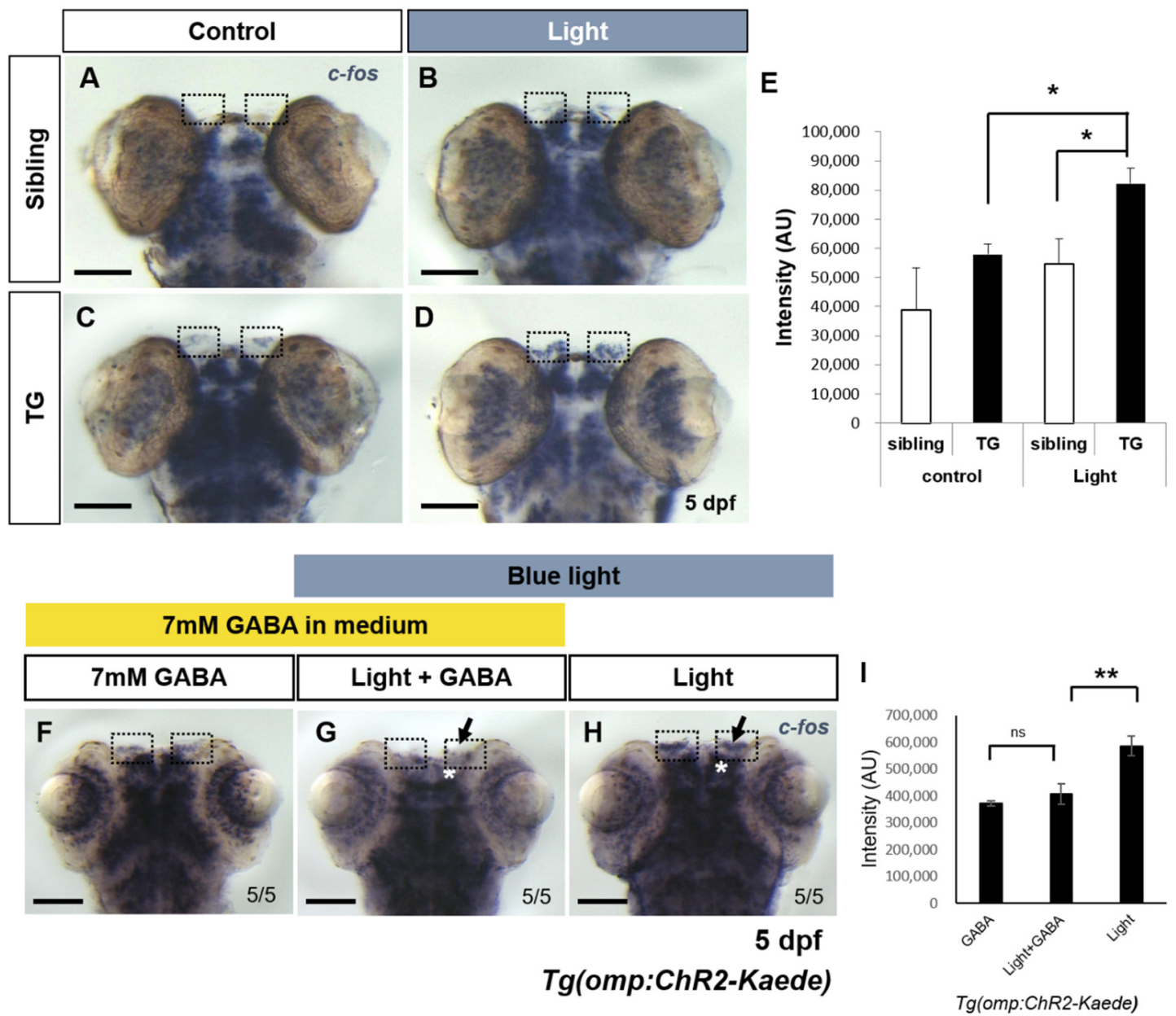

Figure 2. Expression analysis of $c$-fos for evaluating light-induced neuronal activity in transgenic fish. (A-D) Whole-mount in situ hybridization of $c$-fos in the transgenic fish and non-transgenic siblings at $5 \mathrm{dpf}$. (A) Control non-transgenic fish. (B) Light-irradiated non-transgenic fish. (C) Control transgenic fish. (D) Light-irradiated transgenic fish. Dotted boxes indicate olfactory epithelium of $5 \mathrm{dpf}$ zebrafish. In light-irradiated transgenic fish, $c$-fos expression was ectopically induced at the olfactory epithelium (D). (E) Quantitative analysis of $c$-fos expression in olfactory epithelium. Intensity of $c$-fos transcripts in olfactory epithelium (dotted boxes) was measured by Image J. $n=4$ for control sibling; $n=4$ for control TG; $n=4$ for light-irradiated sibling; and $n=5$ for light-irradiated TG. (Tg light vs. Tg control, $p=0.0095$; Tg light vs. sibling light, $p=0.04181)$. (F-H) Suppression of light-evoked odor response by GABA application in transgenic fish expressing ChR2 in the olfactory sensory neurons. (F) 7mM GABA treated control transgenic zebrafish at 5 dpf. (G) Optically stimulated transgenic zebrafish with GABA. (H) Light-irradiated transgenic zebrafish. Black arrow indicates olfactory epithelium and asterisk marked olfactory bulb. (I) Intensity of $c$-fos expression in olfactory epithelium upon GABA treatment. $n=5$ for each experiment. (Tg light vs. Tg light + GABA, $p=0.005$ ). All values are represented as mean \pm S.E.M; ${ }^{*} p<0.05$ and ${ }^{* *} p<0.005$. $p$ value was determined by unpaired $\mathrm{t}$ test. ns, not significant. Scale bars: $100 \mu \mathrm{m}$.

\subsection{Light-Driven Neuronal Activity Analysis in the Olfactory Brain Area Using a Genetically Encoded Calcium Indicator}

Intracellular calcium transients reflect the electrical events in neurons, and calcium imaging with calcium indicators is routinely used in neuroscience to assess neuronal activity. To observe neuronal activity triggered by ChR2 specific blue light in central nervous system responsible for olfactory stimulus processing in the transgenic fish, we used a genetically encoded calcium indicator (GECI), GCaMP transgenic line. We generated triple transgenic fish Tg(huc:gal4-VP16;uas:GCaMP7a;omp:ChR2-Kaede) to obtain neuronal cell specific GCaMP expression [33,34]. Subsequently, we monitored neuronal activity induced by ChR2 specific blue light in brain areas responsible for olfactory stimulus processing, including the olfactory bulb, telencephalon and habenula in the transgenic fish 
carrying ChR2 (Figure 3). Before blue light activation, calcium signal was found absent in the olfactory bulb (Figure 3A). After blue light irradiation, we observed a GCaMP signal in some cell bodies beside axon terminals of olfactory sensory neurons. These signals were assumed to be in mitral cells in the glomerulus of the olfactory bulb. The signals increased with the course of time (Figure $3 \mathrm{~B}, \mathrm{~B}^{\prime}, \mathrm{B}^{\prime \prime}$ ). In addition, the intensity of green fluorescence of Kaede induced by light decreased in the olfactory epithelium showing activation of the ChR2-Kaede fusion protein. In the telencephalon, axon projections of mitral cells from olfactory bulb to higher-brain centers were observed (Figure S4). This pattern observed in the telencephalon of transgenic fish is similar to that published in a previous study on axon projections of mitral cells [35].
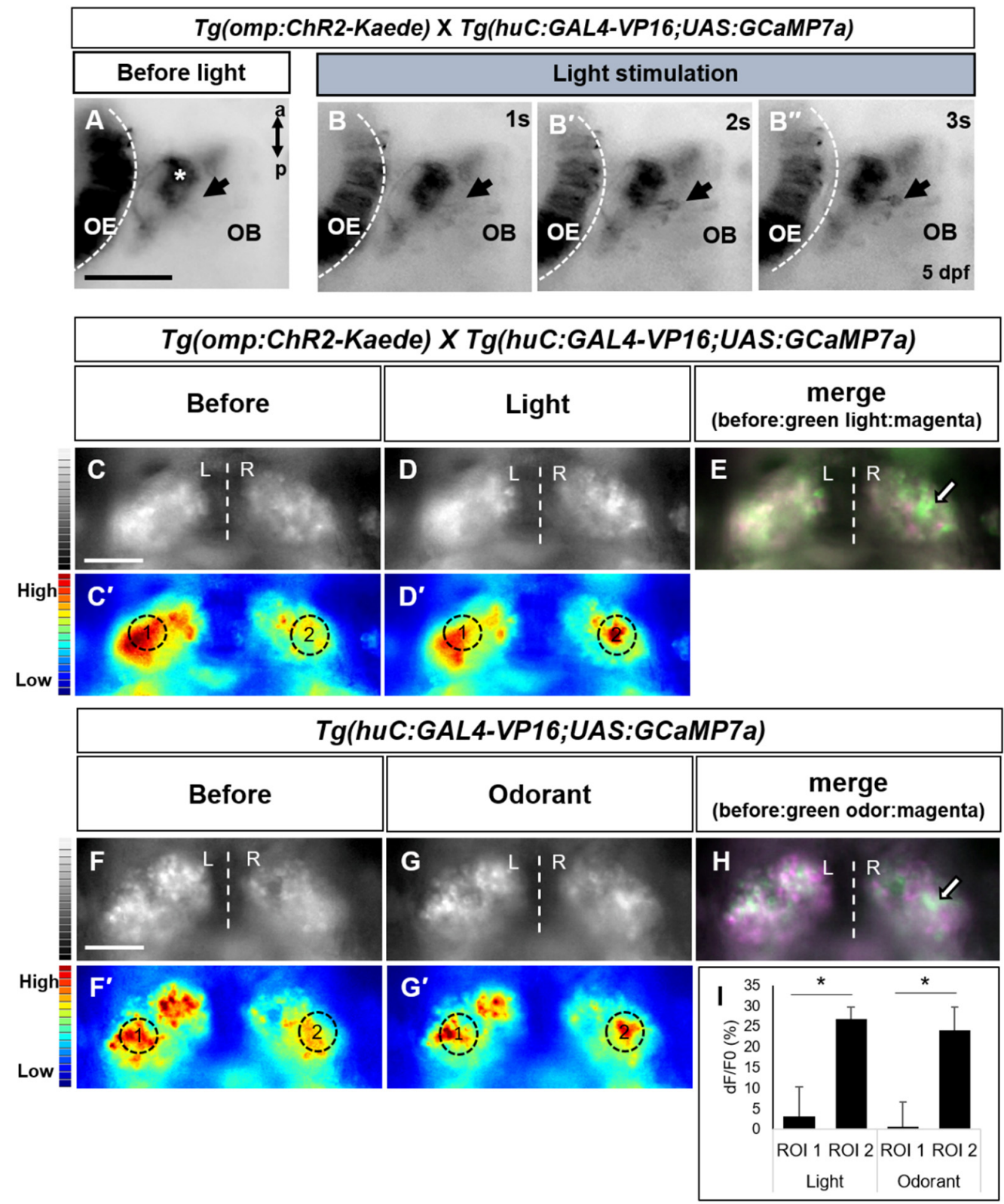

Figure 3. Analysis of calcium transient in olfactory bulb and habenula evoked light/odor stimuli. (A) Olfactory epithelium (OE) and olfactory bulb (OB) of transgenic fish before light irradiation. $\left(\mathbf{B}, \mathbf{B}^{\prime}, \mathbf{B}^{\prime \prime}\right)$ After light stimulation, calcium signals were detected in olfactory bulb. Asterisk indicates axon terminals of olfactory sensory neurons and arrow indicates newly emerging cell bodies by optical stimulation. a, anterior; p posterior. Scale bar: $50 \mu \mathrm{m}$. (C-H) Odor responses of habenula in 
transgenic zebrafish lines. (C-D') In Tg(omp:ChR2-Kaede);Tg(huc:GAL4-VP16;uas:GCaMP7a), habenular neuron responses to light. (F-G') Odorant evoked habenular response in Tg(huc:GAL4VP16;uas:GCaMP7a). Cells before stimulated were colored in magenta, and cells responding to light/odor were colored in green $(\mathbf{E}, \mathbf{H})$. Data are representative of at least two independent experiments. (I) Bar chart showing average dF/F0 of ROI 1 (left habenula) or ROI 2 (right habenula) in zebrafish brain expressing GCaMP7a upon the ChR2 driven light or odor activation ( $n=3$ each group). Dotted circles depict ROI of quantitative analysis. All values represent mean \pm S.E.M. * $p<0.05$. $p$ value was determined by Student's $\mathrm{t}$ test. $\mathrm{L}$, left; $\mathrm{R}$, right; ROI, region of interest. Scale bars: $50 \mu \mathrm{m}$.

The habenula is a small, complex, and evolutionary conserved brain structure located at the dorsal end of the diencephalon. The habenula is composed of two parts based on connectivity and functional heterogeneity. The medial and lateral habenulae in mammals are homologous to the dorsal and ventral habenulae of fish, respectively. The innervation of mitral cell into the right habenula in zebrafish also has been reported [36-39]. We monitored neuronal activity of habenulae in the Tg(omp:ChR2-Kaede) crossed with $T g$ (huc:GAL4; $u a s: G C a M P 7 a$ ) transgenic fish. First, strong and spontaneous activity of GCaMP was detected throughout the habenula compared to the other brain region in the transgenic fish (Figure $3 C-G^{\prime}$ ). The spontaneous activity in these areas is believed to be associated with numerous behavioral functions, including sensory processing, learning and sleeping [40]. We observed calcium transients of the right side of habenula when light was irradiated (Figure 3D, $\mathrm{D}^{\prime}$ ) consistent with the innervation of mitral cells into the right habenula [39]. Left-Right (LR) asymmetries in habenular nuclei coupled with their asymmetric afferent input may underlie the functional asymmetries. Dreosti and colleagues suggested that most habenular neurons responding to odors are found in the right side of habenula, and disruption of this asymmetry may affect sensory processing [36]. To confirm whether the calcium response in the right habenula was elicited by light-driven odor response, we employed an odorant in the Tg(huc:GAL4-VP16;uas:GCaMP7a) fish. We examined whether the odor response could recapitulate the same calcium transient pattern in habenular neurons. As in the optically stimulated Tg(omp:ChR2-Kaede) fish, similar pattern of calcium transient that was showed in the right habenula was observed in the odorant treated Tg(huc:GAL4-VP16;uas:GCaMP7a) fish (Figure 3G, G'). Although the size and shape of the habenula was slightly different, the right side of habenula was activated in both light-illuminated transgenic fish and odorant treated fish. Moreover, the activated region of habenular neurons was located more dorsally. Through a more detailed comparison, we observed that the activated region of habenula was similar (Figure 3E,H and Figure S5). Additionally, we found that the $\mathrm{dF} / \mathrm{F0}$ at the right habenula region (ROI 2) was similar in ChR2-light activated transgenic fish and odor activated fish. Compared to this, the $\mathrm{dF} / \mathrm{F} 0$ of the left habenula (ROI 1) was significantly lower than right habenula (Figure 3I). The activated region showed consistency with previous findings that showed olfactory stimulus induced responses in the right habenula $[36,39,40]$. The slight variability of habenula and positions of activated neurons was likely due to developmental variability across individual zebrafish.

\subsection{Behavioral Adaptation to Light Evoked Odor Response in the Transgenic Zebrafish}

Next, we analyzed the behavior of our transgenic fish to determine how photoactivation of OSNs affects behavioral aspects of zebrafish larvae. Sensory information is abundant in the environment of zebrafish. Distinct brain regions receive different sensory information and integrate it to allow appropriate behavioral responses. For example, olfactory stimulation is known to regulate processing of visual signals [41]. However, when animals receive conflicting sensory cues such as aversive odors and appetitive light cues, mechanisms underlying the animal's behavioral responses are not fully understood. In previous experiments, we found an aroma oil that zebrafish larvae avoided in a crossshaped maze. Additionally, zebrafish larvae showed phototaxis, swimming toward a light source, in light-dark choice task [8-10]. Combining these opposing sensory cues, 
we designed a behavioral experiment as shown in Figure 4. In this experiment, both wild type and transgenic zebrafish larvae swam toward the blue light-illuminated side (Figure 4B). When we administered an aversive odorant (Citrus paradisi) for $5 \mathrm{~min}$, both wild type and transgenic fish swam away from the olfactory cue (Figure 4G). When we administered the odorant and blue light together, wild type fish swam away from the arm where these cues were presented (Figure $4 \mathrm{C}, \mathrm{H}$ ). This suggests that the wild type zebrafish avoid the aversive odorant despite the conflict due to their innate phototaxis. In contrast, the transgenic fish did not show preference for either the light or the odor cue (Figure $4 \mathrm{E}, \mathrm{H}$ ). Whether this alteration of behavior is due to processes similar to those underlying olfactory adaptation [42] is an empirical question that requires experimental analyses in the future. Adaptation to odors is known to reduce perception of an odor by repeated stimulation [42]. Taken together, these results suggest that optical stimulation of OSNs in transgenic zebrafish could alter the behavior of zebrafish larvae. In addition, we showed that an aversive olfactory cue can drive the behavior of zebrafish dominating over phototaxis, and this response can be altered by prolonged olfactory stimulation, likely due to olfactory adaptation.
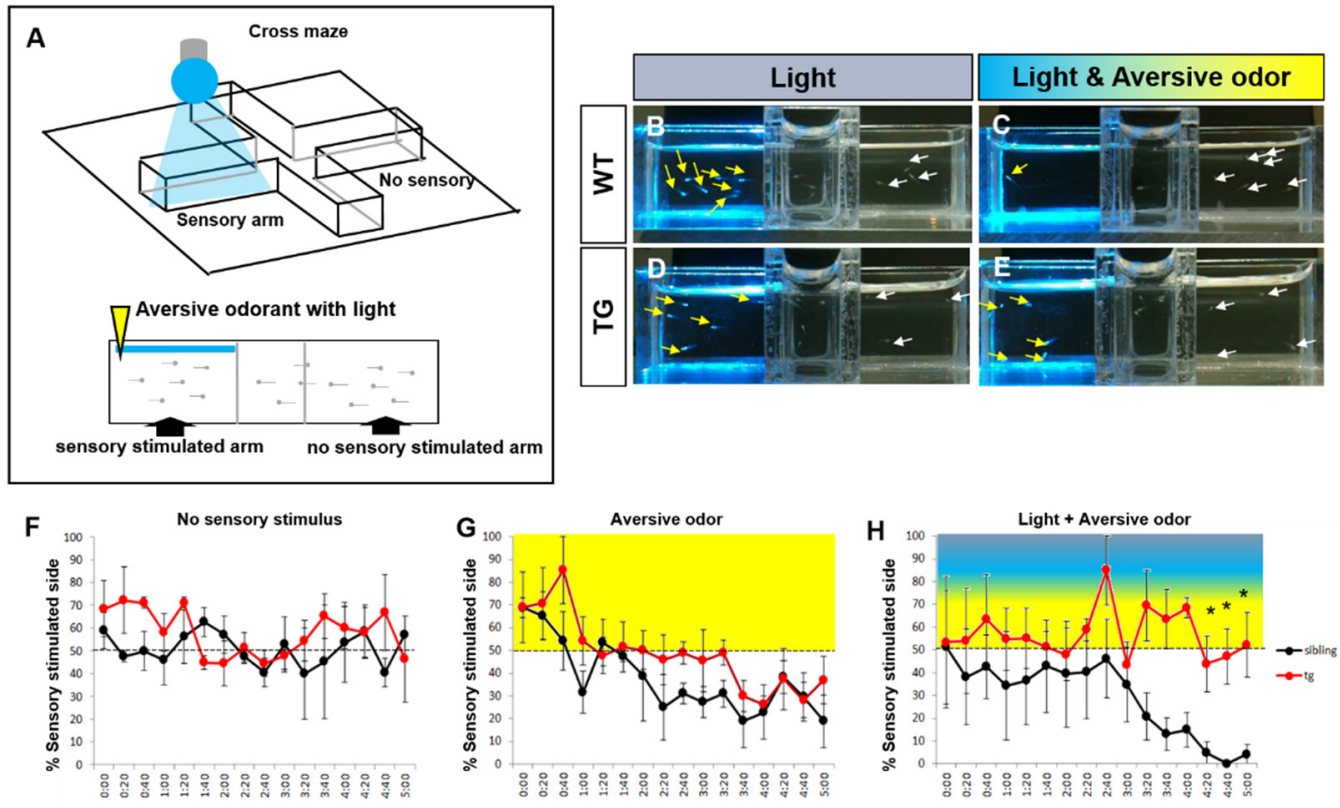

Figure 4. Light and odorant-driven behavior in transgenic zebrafish. (A) Schematic diagram of the conflicting sensory cues-based behavioral test. In a cross-shaped maze, blue light illumination and odorant treatment was performed. The light and the odor cue were both presented in the same (left) arm. (B-E) Images of zebrafish in the behavioral apparatus. The control sibling fish swam toward the blue light illuminated site (B), but when the light was co-presented with the aversive odor cue, they swam away from the stimulus presentation arm (C). The transgenic fish showed no avoidance behavior when the blue light and aversive odor were co-presented (E). Yellow arrows indicate the zebrafish larvae in the stimulus arm; white arrows depict the zebrafish in the no stimulus arm. (F-H) Behavioral response to blue light and aversive odor was measured by the percent of larvae counted in stimulus arm of the cross-shaped maze over time (20 fishes). (F) No sensory stimulus. (G) The aversive odor alone. (H) Blue light and aversive odor co-presented. Dotted lines indicate $50 \%$ of the number of fish in the stimulus arm (chance level performance). All values represent mean \pm S.E.M. ${ }^{*} p<0.05 . p$ value was determined by Student's $t$ test. $n=3$ clutches from three independent experiments.

\section{Discussion}

Optogenetic methods allow activation/deactivation of specified neuronal subpopulations and the monitoring of neuronal activity in the intact brain, methods that help us understand how changes in the activity of specific neuronal circuits contribute to behavior. We employed these tools and generated a transgenic zebrafish model expressing the ChR2-Kaede fusion protein in the olfactory sensory neurons to understand the function 
of neural circuits involved in olfactory processing in zebrafish. The advantage of this optogenetic technique is the non-invasive application of photo-activation which allows specific stimulation of OSNs within intact neuronal networks. Because the larval zebrafish is transparent and displays a rich repertoire of behaviors, we could combine optogenetic methods to activate OSNs and behavioral analysis. In the invertebrate Drosophila model system, optogenetic approaches have been already well established to manipulate the activity of specific OSNs and odor-guided behaviors [43]. However, there are few studies in zebrafish olfactory research using optogenetic applications. In line with this, we successfully established a new transgenic zebrafish larval model allowing manipulation of the activity of OSNs by light. In the current study, we used omp promoter, which is expressed specifically in ciliated OSNs-one of several types of OSN; others include microvillous OSNs which are distinct from ciliated one in zebrafish [21]. Since we have activated only ciliated OSNs in this study, it remains to be investigated which odorant receptor contributes to olfactory behaviors by using a specific promoter of distinct olfactory receptor.

Immediate early genes (IEGs) respond rapidly and transiently to a variety of cellular stimuli [26,44]. Brain mapping using $c$-fos mRNA has been often employed in neuroscience research $[8,45]$. Here, we analyzed light-induced neuronal activity in olfactory epithelium by quantifying the expression of an immediate early gene, $c-f o s$, demonstrating that our system effectively activates OSNs. The blue light-evoked neuronal activity in transgenic fish was repressed in the olfactory epithelium and olfactory bulb by administration of the inhibitory neurotransmitter GABA. These results suggest that neuronal activity by light stimulation was regulated by the GABAergic system which influences odor-evoked activities [31,32].

To monitor activity of central brain areas involved in olfactory stimulus processing more precisely, we used a genetically encoded calcium indicator, GCaMP. We observed calcium transients in the mitral cell region of the olfactory bulb and in the olfactory tract of telencephalon region. Additionally, the right habenular neurons responded to blue light in the transgenic fish carrying ChR2. When an odorant was administered in the Tg(huc:GAL4VP16; uas:GCaMP7a), similar neuron population in the habenula showed calcium signals by GCaMP. The activated neuronal population was observed in the right dorsal habenula. These findings were consistent with recently reported results about the zebrafish habenula: olfactory mitral cell axons innervate into the right habenula and the dorsal habenula is known to receive various sensory inputs including olfactory stimuli $[35,36,39]$. The habenula has an asymmetric structure and is divided into medial/dorsal and lateral/ventral sections both, in mammals and in fish. The left-right differences of this bilateral brain structure are associated with distinct neuronal populations belonging to specific neurotransmitter systems leading to functionally distinct roles the left vs the right habenula plays [37-40]. When the asymmetry was disrupted, the sensory processing function of the habenula was found to be impaired $[30,36]$. Our results on the blue light-evoked olfactory responses in the transgenic fish through GCaMP in right and dorsal habenular nuclei are consistent with the above-discussed previous findings. However, these analyses of the habenulae could not distinguish whether the responses were aversive or appetitive because the categorization of olfactory information such as attractive vs aversive olfactory cues already starts in the olfactory bulb [40]. Our c-fos and GCaMP analyses suggest that the blue light irradiation to transgenic fish could evoke neuronal activity in the olfactory epithelium and olfactory related brain regions including mitral cells, habenula.

We next asked whether olfactory stimuli by light influences the behavior of zebrafish larvae. In our behavioral analysis, we utilized the known fact that zebrafish larvae exhibit phototaxis. We wanted to contrast this appetitive cue (light) with aversive olfactory cue to examine how manipulation of the olfactory system using optogenetic methods may alter stimulus preference, that is, decision making to go towards or away from a particular stimulus. The optogenetic method allowed us to use light both as an olfactory system controlling tool and also as a stimulus processed by the visual system. This behavioral analysis provides insights on how animals choose when conflicting sensory cues are present. 
In addition, the observation showing the aversive olfactory stimulus to be the most salient cue for decision making in zebrafish suggests that aversive cues are prominent when sensory modalities conflict.

Our combinatory analyses of photoactivation and odorant application in the transgenic and wild type zebrafish emphasize the importance of olfaction. Photoactivated transgenic fish showed less sensitivity compared to wild type, as desensitization occurs naturally upon prolonged exposure to the same olfactory cue [42,46]. One possible explanation for this result may be the cross talk between systems processing stimuli of different modalities in the brain. For example, olfactory stimuli can alter the processing of visual signals in the retina via neuronal projections linking the olfactory bulb to the retina, known as the olfactory-retinal circuit (ORC). Additionally, olfactory stimuli have been found to alter visual processing via regulation of concentration of dopamine in the retina [41,47].

The olfactory marker protein (omp) promoter was used to drive widespread expression of ChR2-Kaede in ciliated olfactory sensory neurons of zebrafish in this study. The opticalactivation of the wide-ranging expression of ChR2-Kaede in olfactory sensory neurons could elicit the olfactory driven neuronal activity in olfactory epithelium, olfactory bulb and habenula. Nevertheless, whether the olfactory response was attractive or aversive could not be determined in the transgenic zebrafish. Because recognition and discrimination of odor depends upon the pattern of activation of the odorant receptors in OSNs [48], the global activation of OSNs by light in the transgenic fish is expected to be difficult in terms of motivational value in behavioral assays.

In conclusion, we established a transgenic model to manipulate and monitor the effects of olfactory stimuli in living zebrafish. Analysis of the expression of an immediate early gene and using a transgenic approach for a calcium indicator, GCaMP, allowed us to evaluate neuronal activity changes induced by light in the olfactory stimulus processing system of the transgenic fish and also helped us study correlated behavioral responses. Taken together, our results suggest that the transgenic system employed here will be appropriate to study the neural circuit of olfactory behavior of zebrafish. Given the translational relevance of the zebrafish, our pioneering work may lead to better understanding of the neurobiological mechanisms of olfactory stimulus processing in other vertebrates as well.

\section{Materials and Methods}

\subsection{Animals}

Wild-type zebrafish (Danio rerio), outbred in our fish facility, were maintained at $28.5{ }^{\circ} \mathrm{C}$ in a 14/10 h light and dark cycle. The Animal Ethics Committee of Chungnam National University (CNU 00191) approved all animal experiments. Embryos were raised in embryo medium at $28.5^{\circ} \mathrm{C}$ and embryonic stages were determined by standard method [49]. To inhibit melanization of embryos, phenylthiourea (1-phenyl 2-thiourea, PTU, Sigma, St. Louis, MI, USA) was administered at $10 \mathrm{hpf}$ (hours post fertilization). Zebrafish were obtained from the Zebrafish Center for Disease Modeling.

\subsection{Establishment Transgenic Zebrafish for the Omp-Driven ChR2-Kaede}

\subsubsection{Construction of Expression Vector}

To express ChR2 in zebrafish olfactory sensory neurons, firstly we isolated $5^{\prime}$ upstream promoter region and $3^{\prime}$ untranslated region (UTR) of the zebrafish olfactory marker protein, omp (AB073551) from the Pomp-GFP expression vector (a gift from Tomoyuki Yoshida, University of Tokyo, Tokyo, Japan) [22]. The $2.7 \mathrm{~kb}$ of promoter region and the $3 \mathrm{~kb}$ of $3^{\prime}$-untranslated (UTR) region were amplified by PCR following primers. omp promoter forward primer5'-GCG TCG ACT GTT TTG GTG GCA GAT CTC G-3' omp promoter reverse primer $5^{\prime}$-CGG ATC CTG CGT CTA CTG TGT GTC CTT GG-3'; omp $3^{\prime}$ UTR forward primer 5'-TGG GAA TTC GAG AAA ATG AAG GGT ACA ACG G-3' omp 3'UTR (reverse) 5'-GTC TAG ATG TCA GAA GTG CTT GCT AAG GCT-3'. The primers were designed by using zebrafish genome database (http:/ / www.ensembl.org, ensemble ID: ENSDARG00000032380) and containing some restriction enzyme recognition 
sequences and the PCR products were subcloned into expression vector, mini-Tol2 EGFP. To express ChR2 and Kaede fused protein in zebrafish olfactory sensory neurons, the primers were designed to insert specific restriction enzyme recognition (cutting) sites for Kaede (AB085641) [23,24], amplified by PCR and subcloned in-frame into C-terminus of ChR2 (AF461397) in pPD96.52 vector using Kpn I and EcoR I (Takara Bio Inc., Shiga, Japan) restriction enzymes [18,50]. pPD96.52_ChR2-YFP vector was provided by Prof. Junho Lee [50]. Kaede forward primer (5'-TCG GCC AGG TAC CGA TGA GTC TGA T-3') containing Kpn $I$ and reverse primer (5'-TTG AAT CCC TAC TTG ACG TTG TCC GGC A-3') containing $E c o R I$. The fusion nucleotide was cut with BamH I and EcoR I subcloned into mini-Tol2 expression vector containing omp promoter and $3^{\prime}$ UTR.

\subsubsection{Microinjection and Generation of Transgenic Zebrafish Lines}

Zebrafish embryos were injected with Tol2-based expression construct and Tol2 transposase mRNA at 1-cell stage. To generate stable transgenic line, injected embryos were raised to adulthood. The injected fish were crossed with wild type fish and the offspring were screened for Kaede expression using fluorescence microscopy (Leica MZ16FA, Wetzlar, Germany).

\subsection{Whole-Mount In Situ Hybridization}

Immediate early gene, $c$-fos (NM_205569) was amplified by RT-PCR using the following primers (5'-GGG ATC CGA CAG GAT GAT GTT TAC CAG CCT T-3' and $5^{\prime}$-CCT CGA GGT GGA ATC TCA AAG AGT GAG GAG G-3'). The PCR product was cloned into the pGEM-T-easy vector (Promega, Madison, WI, USA). To synthesize RNA probe, pGEM-Teasy vector containing $c$-fos was linearized with Spe I restriction enzyme. The linearized fragment was transcribed in vitro using T7 RNA polymerase (Thermo scientific, Waltham, MA, USA) and digoxigenin-labeled UTP (Roche, Basel, Switzerland). Whole-mount in situ hybridization was performed as previously described [51] with the anti-sense probe. To examine $c$-fos expression, embryos were fixed 30 min after 5 s-light irradiation with maximal intensity of the mercury lamp (DM 5000B, Leica, Wetzlar, Germany).

\subsection{Light Stimulation and Calcium Imaging}

To visualize calcium activity of light-driven olfactory stimulation in this transgenic system, we used transgenic zebrafish Tg(huc:GAL4-VP16) [33] and Tg (uas:GCaMP7a) [34]. These transgenic lines were obtained from the Koichi Kawakami lab through the central facility of NBRP Japan (RIKEN). The Tg(omp:ChR2-Kaede) fish were crossed with Tg(huc:GAL4;uas:GCaMP7a). The triple transgenic zebrafish of 4-6 dpf were embedded in 5\% methylcellulose (Sigma Chemical Co., St. Louis, MO, USA, M0512) in embryo medium (sea salt 0.006\% Sigma, S9883). Each embryo was irradiated using a Leica DM 5000B upright compound microscope and $20 \times / 0.5$ NA objective equipped with a mercury lamp using a GFP filter (480nm). To target olfactory epithelium region, diaphragm was used. Odorant Citrus paradisi aroma oil was used in this experiment to monitor habenular activity. Calcium activity was observed using a fluorescence microscope (DM 5000B, Leica, Wetzlar, Germany) equipped with a CCD camera (DC300 FX, Leica, Wetzlar, Germany). Due to spectral overlap and subsequent cross-talk, the use of ChR2 specific blue light and GCaMPaided imaging represents a problem. To minimize ChR2 activation during calcium imaging, we irradiated the olfactory epithelium first, and subsequently we adjusted the position of larvae to avoid direct olfactory region irradiation, which allowed us to focus our imaging on the other brain regions. To calculate $\mathrm{dF} / \mathrm{F} 0$ we first measured the average intensity of GCaMP7a in the ROI 1 (left habenula) and ROI 2 (right habenula), before ChR2-light or odor activation as baseline using ImageJ (National Institute of Health, Bethesda, Maryland, MD, USA). After activation, we measured the average intensity and then we calculated $\mathrm{dF} / \mathrm{F} 0$ by dividing intensity change (activation-before) by baseline intensity. 


\subsection{Behavioral Assay Odorant Treatment}

In a cross-shaped maze (width $12.5 \mathrm{~cm} \times$ height $8.5 \mathrm{~cm}$, maze width $1 \mathrm{~cm}$ ), 20 zebrafish larvae $5 \mathrm{dpf}$ of age were placed. Then Citrus paradisi aroma oil was treated with DMSO (dimethyl sulfoxide, Junsei Chemical Co.,Ltd., Tokyo, Japan) with 1:1 volume ratio. The left arm (stimulus arm) of the cross maze with freely moving zebrafish larvae were irradiated using fluorescence microscope (Leica MZ16FA, Wetzlar, Germany) equipped with a mercury lamp using a GFP filter $(480 \mathrm{~nm}) .2 \mathrm{uL}$ of odorant (Citrus paradisi aroma oil) was slowly injected into the end of the stimulus arm. Upon stimulus presentation, the behavior of zebrafish larvae was recorded from the side view using a video camera (Sony, HDR-CX190, Tokyo, Japan). During 5-min-long behavioral test sessions, the number of fish in each arm (stimulus, $\mathrm{S}$ and no stimulus, $\mathrm{N}$ ) was counted with $20 \mathrm{~s}$ interval.

Supplementary Materials: The following are available online at https:/ /www.mdpi.com/article/10 $.3390 /$ ijms22137191/s1.

Author Contributions: Conceptualization, C.-H.K. and Y.-M.J.; data analysis, Y.-M.J., J.-S.L., K.-S.H. and T.-I.C.; funding acquisition, C.-H.K. and R.G.; methodology, Y.-M.J. and T.-I.C.; writing and editing, Y.-M.J., C.-H.K. and R.G. All authors have read and agreed to the published version of the manuscript.

Funding: This work was supported by grants from the National Research Foundation (NRF) of Korea (2018M3A9B8021980, 2020R1A5A8017671). R.G. was supported by the Distinguished Professorship Award by the University of Toronto Mississauga.

Institutional Review Board Statement: The study was conducted according to approved guidelines and regulations of the Institutional Animal Care and Use Committee at the Animal Ethics Committee of Chungnam National University (Approval number: CNU 00191).

Informed Consent Statement: Not applicable.

Data Availability Statement: The data presented in this study are available on request from the corresponding author.

Conflicts of Interest: All authors declare no conflicts of interest.

$\begin{array}{ll}\text { Abbreviations } \\ \text { ChR2 } & \begin{array}{l}\text { Channelrhodpsin } \\ \text { olfactory marker protein }\end{array} \\ \text { omp } & \begin{array}{l}\text { gamma-Aminobutyric acid } \\ \text { GABA }\end{array} \\ \text { GECI } & \text { genetically encoded calcium indicator } \\ \text { OSNs } & \text { olfactory sensory neurons }\end{array}$

\section{References}

1. Song, Y.H.; Kim, J.H.; Jeong, H.W.; Choi, I.; Jeong, D.; Kim, K.; Lee, S.H. A Neural Circuit for Auditory Dominance over Visual Perception. Neuron 2017, 93, 1236-1237. [CrossRef] [PubMed]

2. Horberg, T.; Larsson, M.; Ekstrom, I.; Sandoy, C.; Lunden, P.; Olofsson, J.K. Olfactory Influences on Visual Categorization: Behavioral and ERP Evidence. Cereb. Cortex 2020, 30, 4220-4237. [CrossRef]

3. Yoshihara, Y. Molecular genetic dissection of the zebrafish olfactory system. Results Probl. Cell Differ. 2009, 47, 97-120.

4. Whitlock, K.E. The sense of scents: Olfactory behaviors in the zebrafish. Zebrafish 2006, 3, 203-213. [CrossRef]

5. Kermen, F.; Darnet, L.; Wiest, C.; Palumbo, F.; Bechert, J.; Uslu, O.; Yaksi, E. Stimulus-specific behavioral responses of zebrafish to a large range of odors exhibit individual variability. BMC Biol. 2020, 18, 66. [CrossRef]

6. Burgess, H.A.; Schoch, H.; Granato, M. Distinct retinal pathways drive spatial orientation behaviors in zebrafish navigation. Curr. Biol. 2010, 20, 381-386. [CrossRef] [PubMed]

7. Mueller, K.P.; Neuhauss, S.C.F. Behavioral neurobiology: How larval fish orient towards the light. Curr. Biol. 2010, 20, R159-R161. [CrossRef]

8. Lau, B.Y.; Mathur, P.; Gould, G.G.; Guo, S. Identification of a brain center whose activity discriminates a choice behavior in zebrafish. Proc. Natl. Acad. Sci. USA 2011, 108, 2581-2586. [CrossRef] [PubMed]

9. Fernandes, A.M.; Fero, K.; Arrenberg, A.B.; Bergeron, S.A.; Driever, W.; Burgess, H.A. Deep brain photoreceptors control light-seeking behavior in zebrafish larvae. Curr. Biol. 2012, 22, 2042-2047. [CrossRef] [PubMed] 
10. Chen, F.; Chen, S.; Liu, S.; Zhang, C.; Peng, G. Effects of lorazepam and WAY-200070 in larval zebrafish light/dark choice test. Neuropharmacology 2015, 95, 226-233. [CrossRef]

11. Facciol, A.; Iqbal, M.; Eada, A.; Tran, S.; Gerlai, R. The light-dark task in zebrafish confuses two distinct factors: Interaction between background shade and illumination level preference. Pharmacol. Biochem. Behav. 2019, 179, 9-21. [CrossRef]

12. Facciol, A.; Tran, S.; Gerlai, R. Re-examining the factors affecting choice in the light-dark preference test in zebrafish. Behav. Brain Res. 2017, 327, 21-28. [CrossRef] [PubMed]

13. Bogli, S.Y.; Huang, M.Y. Spontaneous alternation behavior in larval zebrafish. J. Exp. Biol. 2017, 220, 171-173. [CrossRef] [PubMed]

14. Roberts, A.C.; Bill, B.R.; Glanzman, D.L. Learning and memory in zebrafish larvae. Front. Neural Circuits 2013, 7, 126. [CrossRef]

15. Deisseroth, K. Optogenetics. Nat. Methods 2011, 8, 26-29. [CrossRef]

16. Portugues, R.; Severi, K.E.; Wyart, C.; Ahrens, M.B. Optogenetics in a transparent animal: Circuit function in the larval zebrafish. Curr. Opin. Neurobiol. 2013, 23, 119-126. [CrossRef] [PubMed]

17. Zhang, F.; Vierock, J.; Yizhar, O.; Fenno, L.E.; Tsunoda, S.; Kianianmomeni, A.; Prigge, M.; Berndt, A.; Cushman, J.; Polle, J.; et al. The microbial opsin family of optogenetic tools. Cell 2011, 147, 1446-1457. [CrossRef]

18. Nagel, G.; Brauner, M.; Liewald, J.F.; Adeishvili, N.; Bamberg, E.; Gottschalk, A. Light activation of channelrhodopsin-2 in excitable cells of Caenorhabditis elegans triggers rapid behavioral responses. Curr. Biol. 2005, 15, 2279-2284. [CrossRef]

19. Douglass, A.D.; Kraves, S.; Deisseroth, K.; Schier, A.F.; Engert, F. Escape behavior elicited by single, channelrhodopsin-2-evoked spikes in zebrafish somatosensory neurons. Curr. Biol. 2008, 18, 1133-1137. [CrossRef]

20. Kravitz, A.V.; Freeze, B.S.; Parker, P.R.; Kay, K.; Thwin, M.T.; Deisseroth, K.; Kreitzer, A.C. Regulation of parkinsonian motor behaviours by optogenetic control of basal ganglia circuitry. Nature 2010, 466, 622-626. [CrossRef]

21. Sato, Y.; Miyasaka, N.; Yoshihara, Y. Mutually exclusive glomerular innervation by two distinct types of olfactory sensory neurons revealed in transgenic zebrafish. J. Neurosci. 2005, 25, 4889-4897. [CrossRef] [PubMed]

22. Yoshida, T.; Ito, A.; Matsuda, N.; Mishina, M. Regulation by protein kinase A switching of axonal pathfinding of zebrafish olfactory sensory neurons through the olfactory placode-olfactory bulb boundary. J. Neurosci. 2002, 22, 4964-4972. [CrossRef] [PubMed]

23. Ando, R.; Hama, H.; Yamamoto-Hino, M.; Mizuno, H.; Miyawaki, A. An optical marker based on the UV-induced green-to-red photoconversion of a fluorescent protein. Proc. Natl. Acad. Sci. USA 2002, 99, 12651-12656. [CrossRef] [PubMed]

24. Moon, H.Y.; Kim, O.H.; Kim, H.T.; Choi, J.H.; Yeo, S.Y.; Kim, N.S.; Park, D.S.; Oh, H.W.; You, K.H.; De Zoysa, M.; et al. Establishment of a transgenic zebrafish EF1alpha:Kaede for monitoring cell proliferation during regeneration. Fish. Shellfish Immunol. 2013, 34, 1390-1394. [CrossRef]

25. Arrenberg, A.B.; Del Bene, F.; Baier, H. Optical control of zebrafish behavior with halorhodopsin. Proc. Natl. Acad. Sci. USA 2009, 106, 17968-17973. [CrossRef] [PubMed]

26. Curran, T.; Morgan, J.I. Fos: An immediate-early transcription factor in neurons. J. Neurobiol. 1995, 26, 403-412. [CrossRef]

27. Kovacs, K.J. Measurement of immediate-early gene activation- c-fos and beyond. J. Neuroendocrinol. 2008, 20, 665-672. [CrossRef]

28. Okuno, H. Regulation and function of immediate-early genes in the brain: Beyond neuronal activity markers. Neurosci. Res. 2011, 69, 175-186. [CrossRef]

29. Bowen, M.T.; Kevin, R.C.; May, M.; Staples, L.G.; Hunt, G.E.; McGregor, I.S. Defensive aggregation (huddling) in Rattus norvegicus toward predator odor: Individual differences, social buffering effects and neural correlates. PLoS ONE 2013, 8, e68483. [CrossRef]

30. DeCarvalho, T.N.; Akitake, C.M.; Thisse, C.; Thisse, B.; Halpern, M.E. Aversive cues fail to activate fos expression in the asymmetric olfactory-habenula pathway of zebrafish. Front. Neural Circuits 2013, 7, 98. [CrossRef]

31. Mack-Bucher, J.A.; Li, J.; Friedrich, R.W. Early functional development of interneurons in the zebrafish olfactory bulb. Eur. J. Neurosci. 2007, 25, 460-470. [CrossRef]

32. Tabor, R.; Yaksi, E.; Friedrich, R.W. Multiple functions of GABAA and GABAB receptors during pattern processing in the zebrafish olfactory bulb. Eur. J. Neurosci. 2008, 28, 117-127. [CrossRef]

33. Kimura, Y.; Satou, C.; Higashijima, S. V2a and V2b neurons are generated by the final divisions of pair-producing progenitors in the zebrafish spinal cord. Development 2008, 135, 3001-3005. [CrossRef]

34. Muto, A.; Ohkura, M.; Abe, G.; Nakai, J.; Kawakami, K. Real-time visualization of neuronal activity during perception. Curr. Biol. 2013, 23, 307-311. [CrossRef] [PubMed]

35. Miyasaka, N.; Morimoto, K.; Tsubokawa, T.; Higashijima, S.; Okamoto, H.; Yoshihara, Y. From the olfactory bulb to higher brain centers: Genetic visualization of secondary olfactory pathways in zebrafish. J. Neurosci. 2009, 29, 4756-4767. [CrossRef] [PubMed]

36. Dreosti, E.; Llopis, N.V.; Carl, M.; Yaksi, E.; Wilson, S.W. Left-right asymmetry is required for the habenulae to respond to both visual and olfactory stimuli. Curr. Biol. 2014, 24, 440-445. [CrossRef] [PubMed]

37. Amo, R.; Fredes, F.; Kinoshita, M.; Aoki, R.; Aizawa, H.; Agetsuma, M.; Aoki, T.; Shiraki, T.; Kakinuma, H.; Matsuda, M.; et al. The habenulo-raphe serotonergic circuit encodes an aversive expectation value essential for adaptive active avoidance of danger. Neuron 2014, 84, 1034-1048. [CrossRef]

38. Viswanath, H.; Carter, A.Q.; Baldwin, P.R.; Molfese, D.L.; Salas, R. The medial habenula: Still neglected. Front. Hum. Neurosci. 2013, 7, 931. [CrossRef] [PubMed]

39. Krishnan, S.; Mathuru, A.S.; Kibat, C.; Rahman, M.; Lupton, C.E.; Stewart, J.; Claridge-Chang, A.; Yen, S.C.; Jesuthasan, S. The right dorsal habenula limits attraction to an odor in zebrafish. Curr. Biol. 2014, 24, 1167-1175. [CrossRef] 
40. Jetti, S.K.; Vendrell-Llopis, N.; Yaksi, E. Spontaneous activity governs olfactory representations in spatially organized habenular microcircuits. Curr. Biol. 2014, 24, 434-439. [CrossRef]

41. Esposti, F.; Johnston, J.; Rosa, J.M.; Leung, K.M.; Lagnado, L. Olfactory stimulation selectively modulates the OFF pathway in the retina of zebrafish. Neuron 2013, 79, 97-110. [CrossRef] [PubMed]

42. Zufall, F.; Leinders-Zufall, T. The cellular and molecular basis of odor adaptation. Chem. Senses 2000, 25, 473-481. [CrossRef]

43. Stortkuhl, K.F.; Fiala, A. The Smell of Blue Light: A New Approach toward Understanding an Olfactory Neuronal Network. Front. Neurosci. 2011, 5, 72. [CrossRef] [PubMed]

44. Kishimoto, N.; Asakawa, K.; Madelaine, R.; Blader, P.; Kawakami, K.; Sawamoto, K. Interhemispheric asymmetry of olfactory input-dependent neuronal specification in the adult brain. Nat. Neurosci. 2013, 16, 884-888. [CrossRef]

45. Milbrandt, J. Nerve growth factor rapidly induces c-fos mRNA in PC12 rat pheochromocytoma cells. Proc. Natl. Acad. Sci. USA 1986, 83, 4789-4793. [CrossRef] [PubMed]

46. Lecoq, J.; Tiret, P.; Charpak, S. Peripheral adaptation codes for high odor concentration in glomeruli. J. Neurosci. 2009, 29, 3067-3072. [CrossRef] [PubMed]

47. Huang, L.; Maaswinkel, H.; Li, L. Olfactoretinal centrifugal input modulates zebrafish retinal ganglion cell activity: A possible role for dopamine-mediated $\mathrm{Ca}^{2+}$ signalling pathways. J. Physiol. 2005, 569, 939-948. [CrossRef] [PubMed]

48. DeMaria, S.; Ngai, J. The cell biology of smell. J. Cell Biol. 2010, 191, 443-452. [CrossRef]

49. Kimmel, C.B.; Ballard, W.W.; Kimmel, S.R.; Ullmann, B.; Schilling, T.F. Stages of embryonic development of the zebrafish. Dev. Dyn. 1995, 203, 253-310. [CrossRef]

50. Lee, H.; Choi, M.-K.; Lee, D.; Kim, H.-S.; Hwang, H.; Kim, H.; Park, S.; Paik, Y.-K.; Lee, J. Nictation, a dispersal behavior of the nematode Caenorhabditis elegans, is regulated by IL2 neurons. Nat. Neurosci. 2012, 15, 107. [CrossRef] [PubMed]

51. Kim, H.T.; Kim, E.H.; Yoo, K.W.; Lee, M.S.; Choi, J.H.; Park, H.C.; Yeo, S.Y.; Lee, D.S.; Kim, C.H. Isolation and expression analysis of Alzheimer's disease-related gene xb51 in zebrafish. Dev. Dyn. 2008, 237, 3921-3926. [CrossRef] [PubMed] 